\title{
Modifiziertes Trachealkanülenmanagement: Platzhaltereinsatz als Option bei erschwerten Dekanülierungen (Pilotprojekt REHAB Basel)
}

\section{Modified Tracheostomy Management: A Protocol for the Application of Stoma Buttons in Difficult Decannulations}

\author{
Autoren \\ U. Frank ${ }^{1,2}$, C. Czepluch ${ }^{1}$, H. Sticher ${ }^{1}$, F. Mätzener ${ }^{1}$, W. Schlaegel ${ }^{1}$, M. Mäder \\ Institute \\ ${ }^{1}$ Schluckzentrum, REHAB Basel, Schweiz \\ ${ }^{2}$ Department Linguistik/kognitive Neurolinguistik, Universität Potsdam
}

\section{Schlüsselwörter \\ - Dekanülierungsmanage- ment \\ - Dilatationstracheostoma \\ - Platzhalter \\ - Respiration \\ - Dysphagie}

Key words

- decannulation protocol

- dilatational tracheostomy

- stoma button

- respiration

- dysphagia

\section{Zusammenfassung \\ $\nabla$}

Tracheotomierte Patienten, die sowohl eine Dysphagie als auch respiratorische Defizite aufweisen, haben nach der Dekanülierung häufig Probleme, sich an die translaryngeale Atmung anzupassen. Wir entwickelten ein Dekanülierungsprotokoll für diese Patientengruppe, das optional in unser bestehendes Trachealkanülenmanagement integriert werden kann. Erfüllt ein Patient die hierfür definierten Kriterien, so erfolgt unter laryngoskopischer Kontrolle die Einlage eines Platzhalters, der bis zu 3 Tage in situ verbleibt. Während dieser Probedekanülierungsphase werden die respiratorischen Funktionen und das Speichelmanagement engmaschig überwacht. Auf der Grundlage dieser Evaluation wird dann die Entscheidung für oder gegen eine endgültige Dekanülierung getroffen. Wir stellen den Ablauf, die Kriterienkataloge und die Evaluationsparameter für diese Probedekanülierungsphase vor und illustrieren den Ablauf anhand von 2 Fallbeispielen.

\section{Abstract \\ $\nabla$}

Current approaches to decannulation management often fail to account for patients with combined swallowing and respiratory deficits. We expanded our existing weaning and decannulation protocol by adding an optional 3-day decannulation trial to evaluate readiness for decannulation. If a patient meets predefined testdecannulation criteria a tracheostomy button is inserted during a laryngoscopic examination and left in situ for up to 3 days. Before, during and after button insertion the patient's respiratory function and saliva management are closely monitored before the decision for or against permanent decannulation is made. We present evaluation criteria, protocols and flow-charts illustrating the 3-day decannulation trial as well as 2 case studies.

\section{Bibliografie}

DOI http://dx.doi.org/ 10.1055/s-0032-1306290

Online-Publikation: 3.7.2012

Rehabilitation 2013;

52: $20-26$

(c) Georg Thieme Verlag KG Stuttgart · New York ISSN 0034-3536

\section{Korrespondenzadresse} Dr. Ulrike Frank

Department Linguistik/ kognitive Neurolinguistik Universität Potsdam

Karl-Liebknecht-Straße 24-25

14476 Potsdam

ufrank@uni-potsdam.de

\section{Einleitung}

Die in den letzten Jahren stetig zunehmende Anzahl von Patientinnen und Patienten mit erworbenen Hirnläsionen und komplexen Schädigungsbildern erfordert spezialisierte Behandlungsmethoden in der neurologischen Frührehabilitation. Dabei ist die Entwicklung qualitativ hochstehender Standards eine wichtige Voraussetzung zur Vermeidung von Komplikationen und zum Erreichen einer bestmöglichen Lebensqualität.

Für eine Tracheotomie und die Versorgung mit einer (geblockten) Trachealkanüle bestehen im Wesentlichen 2 Indikationsbereiche: eine unzureichende Atemfunktion, die durch die Möglichkeit zur transstomatalen Atmung bzw. durch eine maschinelle Beatmung unterstützt werden soll, oder eine schwere Dysphagie mit erheblicher Speichelaspiration, wobei die unteren Atemwege durch die Einlage einer geblockten Trachealkanüle vor dem Aspirat geschützt werden sollen. In der neurologischen Frührehabilitation werden zunehmend tracheotomierte Patienten behandelt, die sowohl respiratorische als auch schluckphysiologische Defizite aufweisen.

Grundsätzlich ist eine möglichst rasche und sichere Dekanülierung primäres Ziel in der Neurorehabilitation tracheotomierter Patienten. Die Ergebnisse vieler Studien zeigen, dass durch die transstomatale Atmung bei Einsatz einer geblockten Trachealkanüle Funktionseinschränkungen entstehen, die einen physiologischen Schluckablauf und reflektorische Schutzfunktionen (z. B. Husten) behindern und zu einer erhöh- 


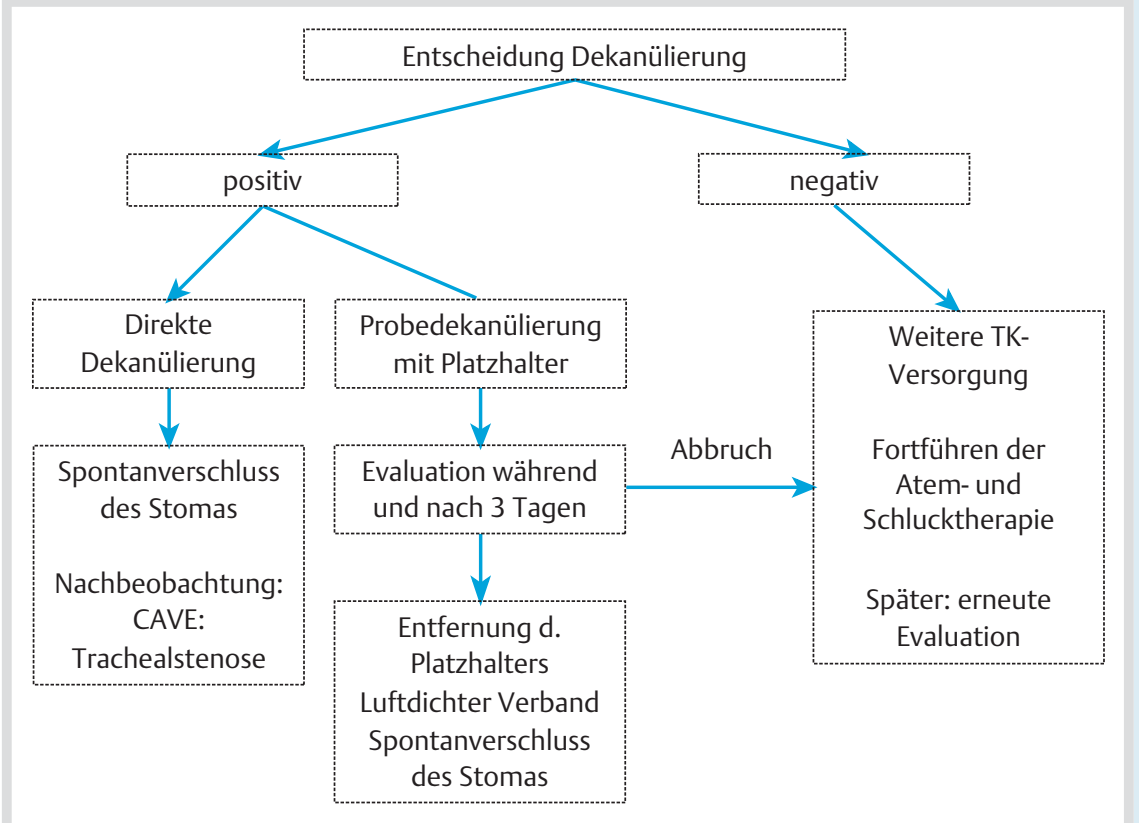

Abb. 1 Basler Dekanülierungsmanagement.

ten Aspirationsrate führen können ([1-3], vgl. aber auch z.B. $[4,5])$. Auch eine korrekt geblockte Trachealkanüle bietet keineswegs einen sicheren Schutz der unteren Atemwege vor aspiriertem Sekret, da es modellbedingt oder durch Druckschwankungen, z. B. beim Husten, zu passagerem „Vorbeiaspirieren“ kommen kann [6].

Im Verlauf der therapeutischen Trachealkanülenentwöhnung $[7,8]$ verbessern die Patienten in aller Regel ihre Fähigkeiten bezüglich ihres aktiven Speichelmanagements, wodurch sich die Aspirationsgefahr reduziert. Im REHAB Basel wird im Rahmen eines interdisziplinären Behandlungsansatzes versucht, den optimalen Zeitpunkt für eine Dekanülierung zu identifizieren. Hierbei müssen die Vorteile einer rechtzeitigen Dekanülierung (z.B. Vermeidung von Funktionseinschränkungen und Langzeitkomplikationen) und die Nachteile einer zu frühen Dekanülierung (bronchopulmonale Komplikationen durch unzureichendes Speichelmanagement) eingeschätzt und abgewogen werden. Als Diskussionsgrundlage dienen fachspezifische Kriterien, die individuell (patientenbezogen) ausgewertet werden [7]. So kann ein Dekanülierungsversuch z. B. auch bei Patienten mit noch unsicherem Speichelmanagement sinnvoll sein, wenn im Verlauf der Entwöhnungsphase die Umstellung auf die translaryngeale Atmung durch Verschluss der entblockten Trachealkanüle gelingt und sich dabei ein positiver Effekt auf die Schluckfunktion und die Schutzmechanismen zeigt.

Eine zentrale Frage der interdisziplinären Diskussion ist, ob der Weg einer temporären oder einer definitiven Dekanülierung beschritten werden soll. Vor allem Patienten mit zusätzlichen respiratorischen Begleiterkrankungen haben häufig Probleme, sich an die translaryngeale Atemarbeit und die veränderte AtemSchluck-Koordination nach erfolgter Dekanülierung anzupassen. In der klinischen Praxis ist nach der Dekanülierung häufig eine „kritische“ Phase von 1-3 Tagen für diese Anpassungsleistung zu beobachten. So zeichnet sich meist schon innerhalb eines Tages eine Dekompensation der erhöhten Atemarbeit mit entsprechenden Stresszeichen (z.B. erhöhte Atemfrequenz, erhöhte Pulsfrequenz, Vigilanzminderung) ab, während sich eine pulmonale Reaktion in Folge von vermehrtem oropharyngealem Aspirat ggf. erst nach 2-3 Tagen manifestiert. Auch kann eine
$\mathrm{CO}_{2}$-Retention erst nach 1-2 Tagen zu einer symptomatischen Hyperkapnie führen. Daher wäre für diese Patienten eine Probedekanülierung optimal, da durch die Möglichkeit einer schnellen Rekanülierung die transstomatale Atmung jederzeit wieder aufgenommen werden kann. Vor allem bei Patienten mit Dilatationstracheostoma ist dies jedoch nicht ohne weiteres möglich, da sich das Stoma nach der Dekanülierung innerhalb kürzester Zeit spontan verschließt. Im Rahmen einer Modifikation des Basler Ansatzes zum Trachealkanülenmanagement wurde daher ein Schema für eine Probedekanülierung unter Verwendung eines Platzhalters entwickelt. Dieses Konzept beinhaltet ein definiertes Ablaufschema sowie Kriterien zur Evaluation der Probedekanülierungsphase. Im Folgenden werden der Ablauf und die Evaluationskriterien vorgestellt und anhand von Fallbeispielen illustriert.

\section{Material und Methoden}

$\nabla$

Eine Probedekanülierung mit Verwendung eines Platzhalters ist im Basler Trachealkanülenmanagement eine Variante für einen Dekanülierungsversuch ( $\bullet$ Abb. 1).

Dieses Vorgehen ist besonders geeignet für Patienten mit Dilatationstracheostoma, die in der Kanülenentwöhnungsphase Schwierigkeiten mit der Anpassung an die translaryngeale Atmung zeigen, häufig verbunden mit ineffektiver Sekretexpektoration. Der Platzhalter soll während der Probedekanülierung das Stoma offen halten, um jederzeit die Möglichkeit einer Rekanülierung zu gewährleisten. Gleichzeitig soll er das Stoma möglichst optimal abdichten, um ein Leaking von Atemluft zu verhindern und eine translaryngeale Atmung zu ermöglichen. Die nachfolgenden Platzhalter werden von uns verwendet.

\section{Platzhaltervariante 1: „Red Button“}

Beim Red Button handelt es sich um einen Silikonplatzhalter, der im Therapiezentrum Burgau entwickelt wird ${ }^{1}$. Er besteht aus einem kurzen kanülenähnlichen Stück aus Vollsilikon und einem

${ }^{1}$ Nähere Informationen zum Red Button: c.gratz@therapiezentrum-burgau.de. 
verstellbaren Schild ( $\bullet$ Abb. 2). Der Außendurchmesser des Red Button entspricht einer Trachealkanüle vom Typ Shiley, Größe 6. Die Länge entspricht der Distanz zwischen Halshaut und Tracheavorderwand, sodass der Button selbst keinen bzw. einen sehr geringen Strömungswiderstand in der Trachea bildet. Durch das Verschieben des gewölbten Schildes kann die Länge variiert werden, sodass eine gute Platzierung im Stomakanal und die Abdichtung des Stomas ermöglicht wird. Da der Red Button ein Vollgussmodell ist, wird auch die Entstehung eines verstärkten Drucks am Stomarand von innen nach außen verhindert. Der Red Button entspricht somit am ehesten den physiologischen Verhältnissen und ermöglicht eine gute Einschätzung der Atemsituation ohne Trachealkanüle. Da der Red Button nicht intratracheal befestigt werden kann, besteht bei Patienten mit kräftigem Hustenstoß jedoch die Gefahr, dass der Button aus dem Stoma disloziert. Ebenso ist der Red Button weniger geeignet für Patienten, die während der Probedekanülierung voraussichtlich tracheal absaugpflichtig bleiben. Diese Patienten werden mit einem Trachea Stent versorgt.

\section{Trachea Stent}

Bei Patienten, die während der Platzhalterphase voraussichtlich weiterhin tracheal abgesaugt werden müssen, verwenden wir einen Tracheostoma Stent (Tracheo Safe, Fa. Fahl), der aus einem Kanülenkörper mit Lumen besteht ( $\bullet$ Abb. 3). Der Stent besteht aus weichem Silikon und ist in Längen von $45 \mathrm{~mm}$ und $70 \mathrm{~mm}$ erhältlich. Das Einführen in den Stomakanal erfordert hier mehr Erfahrung und Geschicklichkeit als beim Red Button. Eine anschließende endoskopische Lagekontrolle ist unerlässlich, da eine Fehlpositionierung zu einer Verengung des subglottischen Raums führen kann. Bei Verwendung des Stent entsteht ein etwas größerer Strömungswiderstand in der Trachea und die Stomaabdichtung kann geringer sein als beim Red Button. Der Stent verfügt am distalen Ende über einen Verschlussdeckel, der einerseits rasch für Absaugung und transstomatale Atmung geöffnet werden kann, andererseits bei Verschluss die transstomatale Atmung unterbindet und eine Beurteilung der translaryngealen Atmung ermöglicht.

\section{Probedekanülierung: Ablauf}

Vor Beginn der Probedekanülierung wird individuell anhand einer Checkliste überprüft, ob die Voraussetzungen für den Platzhaltereinsatz gegeben sind. Es folgt eine bis zu 3-tägige Probedekanülierungsphase. Während der Platzhalterphase werden die respiratorischen Funktionen und das Speichelmanagement engmaschig überprüft. Anhand der Evaluationswerte wird abschließend entschieden, ob eine endgültige Dekanülierung erfolgen kann ( $\bullet$ Abb. 4).

Voraussetzungen für die Verwendung eines Platzhalters Vor Beginn eines Platzhalterversuchs werden die Voraussetzungen hierfür interdisziplinär überprüft. Therapeuten, Pfleger und Ärzte verwenden hierzu eine Checkliste ( $\bullet$ Tab. 1 ), die die Funktionsbereiche Atemfunktion (mit und ohne Trachealkanüle in situ), Speichelmanagement und Husteneffektivität umfasst. Außerdem wird in Abhängigkeit von der voraussichtlichen Absaugpflicht entschieden, ob beim Platzhalterversuch ein Red Button oder ein Trachea Stent verwendet werden soll.

Zusätzlich zu den genannten Kriterien werden patientenbezogene individuelle Stresssymptome definiert und dokumentiert, sodass während der Probedekanülierung Komplikationen erkannt bzw. vermieden werden können. Zusätzlich wird festgelegt, ob

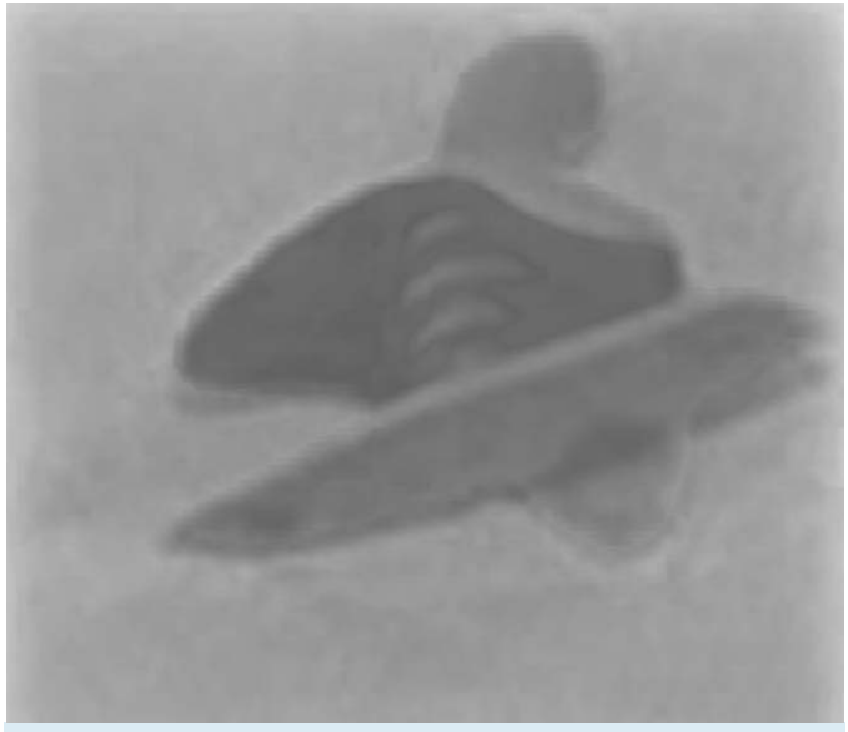

Abb. 2 Red Button (eigene Abbildung, REHAB Basel).

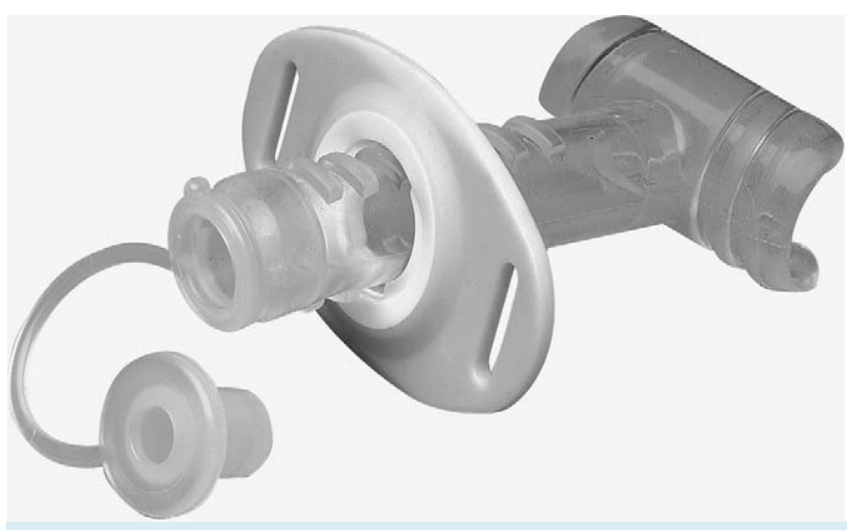

Abb. 3 Trachea Stent (Tracheo Safe, Fa. Fahl Medizintechnik).

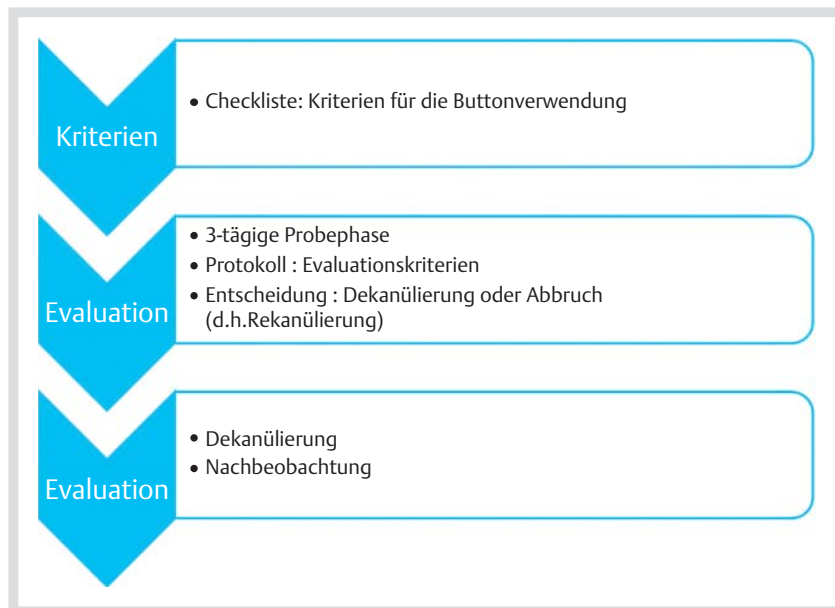

Abb. 4 Flowchart Ablauf Probedekanülierung.

und inwieweit der Patient während des Platzhalterversuchs belastet werden kann, d.h., ob er im üblichen Rahmen an den geplanten Therapien teilnimmt bzw. welche Einschränkungen bestehen. Sofern in die Dysphagietherapie bereits Geschmacksreize in minimalen Mengen einbezogen wurden, wird auf diese Form der Schluckstimulation während des Platzhalterversuchs 
Tab. 1 Checkliste für die Dekanülierung mit Platzhalterverwendung.

\begin{tabular}{|c|c|c|c|c|}
\hline & Funktionsbereich & Beurteilung/Erfassung & JA & NEIN \\
\hline 1 & Atemfunktion (Trachealkanüle in situ) & $\begin{array}{l}\text { - arterielle Blutgaswerte (aBGA): Absolutwerte und Dynamik } \\
\text { - Atemfrequenz in Ruhe: bei geblockter Trachealkanüle } \leq 20 / \text { min } \\
\text { - Lagewechsel zur Beeinflussung der Atemfunktion möglich }\end{array}$ & & \\
\hline 2 & $\begin{array}{l}\text { Atemfunktion (ohne Trachealkanüle) } \\
\text { laryngoskopische } \\
\text { Beurteilung }\end{array}$ & $\begin{array}{l}\text { - ausreichende Ventilationsstellung der Stimmlippen } \\
\text { - Stabilität der Trachea } \\
\text { - Granulationen/Obstruktionen }\end{array}$ & & \\
\hline 3 & Husten & $\begin{array}{l}\text { - Reagibilität } \\
\text { - Fazilitierbarkeit } \\
\text { - Effektivität }\end{array}$ & & \\
\hline 4 & $\begin{array}{l}\text { Speichelmanagement (bei entblockter } \\
\text { Trachealkanüle) }\end{array}$ & $\begin{array}{l}\text { - Schluckreflextriggerung spontan bzw. fazilitierbar } \\
\text { - Schlucksequenz vollständig } \\
\text { - Speicheldrooling durch Lagerung möglich } \\
\text { - Sekretexpektoration (Husten, Ausspucken) möglich }\end{array}$ & & \\
\hline 5 & bei Verwendung des Red Button & $\begin{array}{l}\text { - dichter Verschluss des Stomakanals bis Tracheavorderwand notwendig } \\
\text { - bei motorisch unruhigen Patienten und kräftigem Hustenstoß: Gefahr } \\
\text { der Dislokation } \rightarrow \text { ggf. Entscheidung für Trachea Stent }\end{array}$ & & \\
\hline 6 & bei Verwendung des Trachea Stent & $\begin{array}{l}\text { - Absaugen während der Probedekanülierung voraussichtlich notwendig } \\
\text { - Festlegen der erforderlichen Länge, Durchmesser des Platzhalters }\end{array}$ & & \\
\hline
\end{tabular}

Tab. 2 Zugrunde gelegte Normwerte für die Auswertung der arteriellen Blutgasanalyse (aBGA).

$\begin{array}{ll}\text { ph } & 7,35-7,45 \\ \mathrm{pCO}_{2} & 4,7-6,0 \mathrm{kPA} \\ \mathrm{pO}_{2} & 9,2-15,5 \mathrm{kPA} \\ \mathrm{BE} \text { (base excess) } & -2 \mathrm{bis}+2 \mathrm{mmol} / \mathrm{l} \\ \mathrm{HCO}_{3} \text { (Bikarbonat) } & 21-26 \mathrm{mmol} / \mathrm{l}\end{array}$

verzichtet, um das Risiko eventueller bronchopulmonaler Komplikationen zu minimieren und ggf. Speichelaspirationen sicher einordnen zu können.

\section{Ablauf der 3-tägigen Probephase}

Ein Platzhalterversuch beginnt immer am Wochenanfang, um eine möglichst engmaschige Beobachtung und ggf. eine Verlängerung der Probephase (s. u.) zu gewährleisten. Am Morgen vor der geplanten Platzhaltereinlage werden zunächst die Blutgaswerte $(\mathrm{aBGA})$ bei geblockter Trachealkanüle in situ bestimmt und als Referenzwerte dokumentiert. Als weitere Referenzwerte werden Normwerte von Gesunden ( $\bullet$ Tab. 2) herangezogen. Diese bilden allerdings relative Vergleichswerte, da bei Patienten mit chronischen respiratorischen Erkrankungen (z.B. COPD) individuelle Schwellenwerte anhand des klinischen Verlaufs festgelegt werden müssen.

Der Patient wird dekanüliert und der gewählte Platzhalter eingesetzt. Dies geschieht unter laryngoskopischer Kontrolle, um eine optimale Positionierung des Platzhalters zu gewährleisten und mögliche Obstruktionen im Atemweg auszuschließen. Im Tagesverlauf erfolgt eine engmaschige Überwachung der Atmung, des Sekretmanagements und möglicher Stresssymptome. Diese werden vor der Platzhaltereinlage und im Tagesverlauf (mit eingesetztem Platzhalter) dokumentiert (s. Tab. 3) und dienen als Grundlage für die Entscheidung zum weiteren Vorgehen. Bei positivem Verlauf wird der Platzhalter am Ende des 3. Tages entfernt und das Stoma bis zum Spontanverschluss abgeklebt (entsprechend einer definitiven Dekanülierung). In Einzelfällen kann eine Erweiterung der Probephase auf 5 Tage (oder länger) sinnvoll sein, wenn beispielsweise die Werte stark schwankend und am 3. Tag der Platzhalterphase noch nicht stabil genug sind. Wenn die allgemeinen Abbruchkriterien (siehe nächsten Abschnitt) oder persistierende individuelle Stresssymptome auftreten, wird der Versuch sofort beendet und der Patient rekanüliert. Nach einer weiteren Therapiephase kann dann ein erneuter Dekanülierungsversuch unternommen werden, sofern der Patient die Kriterien hierfür erfüllt.

\section{Kontraindikationen und Abbruchkriterien}

Es bestehen folgende Kontraindikationen für einen Dekanülierungsversuch mit Platzhaltereinsatz:

- instabiler Allgemeinzustand,

- Obstruktion der oberen Atemwege durch ungenügende Ventilationsstellung der Glottis, Stenosen, Granulationen usw.,

- persistierende massive Speichelaspiration ohne Schutzreaktion,

- Materialunverträglichkeit und

- geplante Operation oder Verlegung in der folgenden Woche. Für jeden Patienten gelten generelle und individuelle Abbruchkriterien für die Probedekanülierungsphase. Generelle Abbruchkriterien sind ein signifikanter Anstieg der Pulsfrequenz in Ruhe und ein Anstieg der Atemfrequenz auf $>30 / \mathrm{min}$, die nicht durch therapeutische Maßnahmen wie z.B. Lageveränderungen über einen längeren Zeitraum beeinflussbar sind. Bei einem raschen Abfall der $\mathrm{SpO}_{2}$-Sättigung, der nicht durch aktive Sekretexpektoration oder Absaugen reversibel ist, oder bei akuter Atemnot wird der Platzhalter sofort entfernt und der Patient rekanüliert. Die individuellen Abbruchkriterien werden im Vorfeld interdisziplinär definiert und es wird festgelegt, welche Symptome bzw. welcher Ausprägungsgrad zu einem Abbruch des Platzhalterversuchs führen soll.

\section{Fallbeispiele}

Das Verfahren wurde im Zeitraum von April 2009 bis August 2011 mit 20 Patienten durchgeführt, die die Kriterien für eine Probedekanülierung erfüllten. Es zeigte sich hierbei, dass die Probedekanülierung bei den meisten dieser Patienten zu einer erfolgreichen Dekanülierung führte. Somit konnte in diesen Fällen die Entfernung der Trachealkanüle unter diesen stark kontrollierten Bedingungen früher erfolgen, als dies bei Berücksichtigung konventioneller Dekanülierungskriterien möglich gewesen wäre. Nur in einem Fall wurde die Probedekanülierung abgebrochen und eine Rekanülierung durchgeführt. Dieser und ein 
Tab. 3 Evaluationskriterien während der Probedekanülierungsphase.

$\begin{array}{llll} & \text { Atmungsparameter } & \text { Sekretmanagement } & \text { Stresssymptome } \\ \text { vor Platzhaltereinlage } & - \text { translaryngeale Atmung (Frequenz, } & - \text { Schluckaktivität (Frequenz, } & \text { - Pulsfrequenz } \\ & \text { Atemtiefe) } & \text { Effektivität) } & \text { - individuelle Stresssymptome } \\ \text { während Platzhalterversuch } & -\mathrm{SpO}_{2} \text {-Werte } & - \text { aktive Sekretexpektoration } & \text { - Vigilanz/Wachheit } \\ & -\mathrm{aBGA:} \text { Trachealkanüle in situ vs. } & \text { (Frequenz, Effektivität) } & \\ \text { nach Platzhalterentfernung } & \text { Platzhalter in situ } & - \text { Absaugen von Sekret (Frequenz, } \\ & & \text { Sekretqualität) }\end{array}$

exemplarischer positiver Verlauf sind im Folgenden detaillierter dargestellt.

\section{Patient 1: Erfolgreiche Dekanülierung nach 3-tägiger Probephase}

Patient 1 erlitt 2007 ein schweres Polytrauma mit schwerem Schädel-Hirn-Trauma und Kontusions- und Subarachnoidalblutungen frontal rechts. Nach mehrfachen Shunt-Infekten kam es Anfang 2008 zu einer spontanen intrazerebellären Blutung links. Im Verlauf traten wiederholt epileptische Ereignisse auf. Patient 1 wurde im August 2009 im REHAB Basel aufgenommen; zu diesem Zeitpunkt war seine Vigilanz deutlich eingeschränkt im Sinne eines Minimally Responsive State. Es bestand eine schwere kardiale Begleitsymptomatik mit Vorhofflimmern, außerdem kam es rezidivierend zu Aspirationspneumonien. Logopädisch wurde eine schwere neurogene Dysphagie und eine schwere Kommunikationsstörung diagnostiziert.

Im Verlauf der Trachealkanülenentwöhnung zeigte Patient 1 bei entblockter und verschlossener Kanüle ein deutlich verbessertes Sekretmanagement mit verbesserter Schluckfrequenz und spontaner Sekretexpektoration, die jedoch nicht immer effektiv war. Er benötigte erhebliche Unterstützung bzw. Supervision zur Absicherung der Atmung und der Schluckfunktion während der therapeutischen Entblockungs- und Verschlussintervalle. Aufgrund der positiven Effekte der translaryngealen Atmung auf die Schluck- und Atemfunktion entschieden wir uns 27 Tage nach Beginn der Trachealkanülenentwöhnung trotz des noch unsicheren Speichelmanagements zu einer Probedekanülierungsphase. Bei der aBG-Analyse vor Platzhaltereinlage wich der $\mathrm{pO}_{2}$-Wert $\left(\mathrm{pO}_{2}\right.$ : 7,6 kPA) von den zugrunde gelegten Normwerten ab. Als Platzhalter wurde ein Trachea Stent $(45 \mathrm{~mm})$ verwendet, um ein tracheales Absaugen bei Bedarf zu ermöglichen. Im Verlauf des Tages war kein tracheales Absaugen notwendig. Patient 1 zeigte eine suffiziente translaryngeale Atmung. Der Hustenreflex war spontan auslösbar, die Sekretexpektoration war zunächst noch überwiegend ineffektiv. Die Atemfrequenz lag durchgehend innerhalb des Toleranzbereichs (max. 30/min), die $\mathrm{SpO}_{2}$-Sättigung lag durchgehend bei $>90 \%$. Es zeigten sich Stresssymptome beim Umlagern in Rückenlage, diese relativierten sich bei Seitlagerung. Die erneute Blutgasanalyse wies am Ende des 1. Tages folgende Werte außerhalb des Normbereichs auf: pH 7,5; $\mathrm{BE} 4,0 \mathrm{mmol} / \mathrm{l} ; \mathrm{HCO}_{3}$ $24,7 \mathrm{mmol} / \mathrm{l}$. Im weiteren Verlauf konnte der Patient seine Sekretmengen trotz des eher schwachen Hustenstoßes selbstständig bewältigen. Ein tracheales Absaugen war nicht notwendig.

Die Umstellung auf die translaryngeale Atmung gelang weiterhin problemlos, die Atemfrequenz lag durchgehend bei $<20$ / min, die $\mathrm{SpO}_{2}$-Sättigung bei $>90 \%$. Der Platzhalter wurde am Ende des 3. Tages entfernt und das Stoma abgeklebt. Im Verlauf der folgenden Tage nach endgültiger Dekanülierung zeigte sich deutlich, dass eine unvollständige Stomaabdichtung zu deutlicher Tonuserhöhung, erschwerter Atmung und ineffektiver Se- kretexpektoration führte. Nach vollständiger Stomaabdichtung relativierten sich diese Probleme.

\section{Patient 2: Abbruch des Platzhalterversuchs}

Patient 2 erlitt Anfang 2011 einen Hirnstamminsult mit Wallenbergsyndrom. Es bestanden eine Dysphagie und eine Dysarthrophonie, ein Blicknystagmus nach rechts und Schwindelsymptomatik. Am Tag nach dem Ereignis kam es zu einem akuten respiratorischen Versagen nach Aspiration, sodass Patient 2 zunächst endotracheal intubiert und wenige Tage später tracheotomiert und mit einer geblockten Trachealkanüle versorgt wurde. Es folgte die stationäre Aufnahme im REHAB Basel zur Rehabilitation. Der Vigilanzstatus war unauffällig und der Patient war mit Rollator selbstständig mobil. Schwerpunkte der Rehabilitation waren die Verbesserung der Schluckfunktion und die therapeutische Trachealkanülenentwöhnung. Patient 2 konnte seine Speichelmengen gut ausspucken, die Spontanschluckrate war erheblich reduziert, beim fazilitierten Schlucken benötigte er sehr viel Hilfestellung.

Die Entscheidung für eine Probedekanülierung mit Platzhalter wurde 65 Tage nach Aufnahme getroffen. Nach laryngoskopischer Untersuchung bestand zu diesem Zeitpunkt eine ausreichende Ventilationsstellung beider Stimmlippen. Problematisch war dennoch weiterhin das Speichelmanagement, da Sekret fast ausschließlich durch aktives Ausspucken und nur selten durch Spontanschlucke bereinigt werden konnte. Laryngoskopisch ergab sich kein Hinweis auf Speichelaspiration. Bei entblockter und verschlossener Kanüle wies der Patient eine höhere Spontanschluckrate auf bzw. eine häufigere, wenn auch unvollständige Initiierung der Schlucksequenz. Tagsüber konnte der Patient daher sein Sekret bewältigen, die Kanüle war stundenweise entblockt und verschlossen. Nachts wurde die Kanüle geblockt. Durch den Platzhalterversuch sollte überprüft werden, ob sich das reflektorische Speichelmanagement durch die Dekanülierung konsolidieren lässt. Die Platzhaltereinlage (Tracheo Safe ${ }^{\mathrm{TM}}$ ) erfolgte unter laryngoskopischer Kontrolle.

Der erste Tag und die erste Nacht verliefen komplikationslos. Am Morgen des Folgetages fiel ein in- und exspiratorischer Stridor auf, ein pulmonologisches und HNO-ärztliches Konsil erbrachte keine Klärung der Ursache. Subjektiv ging es dem Patienten gut, insbesondere hatte er keine Atembeschwerden. Eine erneute endoskopische Lagekontrolle des Platzhalters zeigte eine regelrechte Position bei freien Atemwegen. Eine $\mathrm{CO}_{2}$-Retention konnte durch eine aBGA ausgeschlossen werden. Der Platzhalter wurde belassen und der Patient kontinuierlich überwacht. Am dritten Probetag kam es morgens zu einem Sättigungsabfall bis 80\% $\mathrm{SpO}_{2}$ und anschließendem Atemversagen. Über den Platzhalter wurde sehr viel Sekret abgesaugt. Daraufhin wurde der Platzhalterversuch abgebrochen und eine Rekanülierung durchgeführt sowie außerdem eine Atemunterstützung (CPAP) gegeben. Folgende aBGA-Werte lagen zu diesem Zeitpunkt außerhalb der 
Norm: ph 7,28; $\mathrm{pCO}_{2} 8,85 \mathrm{kPa} ; \mathrm{pO}_{2} 8,7 \mathrm{kPa} ; \mathrm{HCO}_{3} 31,1 \mathrm{mmol} / \mathrm{l}$. Eine videofluoroskopische Untersuchung 3 Wochen nach dem Platzhalterversuch zeigt eine auffällige Öffnungsstörung des oberen Ösophagussphinkters (oÖS), die in früheren laryngoskopischen Untersuchungen nicht beschrieben wurde. Im weiteren Verlauf wurde ein plastisches Stoma angelegt; eine Dekanülierung konnte bis zum Zeitpunkt der Entlassung nicht erreicht werden.

Wir vermuten, dass die Öffnungsstörung des oÖS in den Nächten nach der Platzhaltereinlage zu einem pharyngealen Sekretaufstau geführt hat. Tagsüber konnte der Patient seine Speichelmengen aktiv ausspucken, des Nachts war diese Möglichkeit nicht gegeben. Eine nächtliche stille Aspiration scheint wahrscheinlich; in der Folge kam es vermutlich zu einer Verlegung der tieferen Atemwege und somit zu einer sukzessiven Stridorbildung, einem Sättigungsabfall und schließlich zum Atemversagen. Für diese Annahmen spricht auch, dass der Patient bereits vor der Probedekanülierung vermehrt nachts hustete und am ersten Tag der Probedekanülierung sehr häufig produktiv abhustete.

\section{Diskussion}

$\nabla$

Eine Probedekanülierungsphase mit Platzhaltereinsatz ist eine von mehreren möglichen Vorgehensweisen bei der Dekanülierung tracheotomierter Patienten. Sie eignet sich besonders für Patienten mit Dilatationstracheostoma, die gemäß ihres klinischen Verlaufs von einer Dekanülierung wahrscheinlich funktionell profitieren könnten, bei denen jedoch Risikofaktoren bestehen, die eine reguläre Dekanülierung mit spontanem (schnellem) Stomaverschluss erschweren. Die Patientenbeispiele zeigen, dass das hier vorgestellte modifizierte Basler-Dekanülierungsmanagement nicht in jedem Fall eine sichere endgültige Entfernung der Trachealkanüle ermöglichen kann. Vor allem der zweite beschriebene Verlauf verdeutlicht unseres Erachtens die Relevanz einer mehrtägigen Probedekanülierungsphase bei Risikopatienten. Die engmaschige Überprüfung vordefinierter respiratorischer und schluckrelevanter Parameter über einen Zeitraum von mehreren Tagen ermöglicht ein frühzeitiges Erkennen von Risikosituationen und eine entsprechende Intervention während der Probedekanülierungsphase.

Die Behandlung von tracheotomierten Patienten mit Dysphagie gehört mittlerweile zum Alltag der Neurorehabilitation. Umso erstaunlicher ist es, dass bisher nur sehr wenige Publikationen erschienen sind, die über neu entwickelte klinische Ablaufschemata und systematische Evaluationen dieser Ansätze berichten. Die Vorstellung des Basler Ansatzes für eine Probedekanülierungsphase mit Platzhalter soll interdisziplinären Behandlungsteams eine Orientierungshilfe für die Therapie von tracheotomierten Patienten geben. Das hier vorgestellte Vorgehen wurde auf der Grundlage unserer hausinternen Abläufe entwickelt. Diese Abläufe werden in anderen Kliniken zu modifizieren sein und sollten zur Entwicklung eigener interdisziplinärer Hauskonzepte führen.

Die Ergebnisse einer 5-Jahres-Follow-up-Studie zum poststationären Verlauf bei tracheotomierten Patienten [9] verdeutlichen die Relevanz eines fachlichen Austauschs zwischen Behandlungseinrichtungen für tracheotomierte Patienten. So berichtet die Studie, dass Patienten mit Schluckstörungen nach Entlas- sung eine signifikant höhere Mortalitätsrate aufweisen als schluckgesunde Patienten. Bei Analyse der „Entlassungsart“ (Pflegeheim - häusliche Umgebung) und der „Trachealkanülenversorgung“ ( $\mathrm{ja}$ - nein) zeigte sich eine signifikant höhere Mortalitätsrate für die Faktoren „Pflegeheimversorgung“ und „,bestehende Trachealkanülenversorgung“. Dies lässt sicher nicht den Schluss zu, dass in Pflegeheimen generell eine schlechte Versorgung von tracheotomierten Patienten erfolgt. Vielmehr ist nach den Ergebnissen der Studie davon auszugehen, dass bei tracheotomierten Patienten, die in häuslicher Umgebung gepflegt werden, die Indikation für die Kanülenversorgung individueller und häufiger überprüft wird. So war in der Studie eine solche Indikationsüberprüfung das häufigste Anliegen bei Inanspruchnahme einer poststationären Beratung, die allen untersuchten Patienten angeboten wurde. Diese Reevaluation der Indikationsstellung findet bedauerlicherweise nicht regelhaft, sondern meist auf Betreiben der Betroffenen bzw. deren Angehörigen statt. Dabei sollten das erhöhte Risiko und das erforderliche Monitoring, die mit einer Dekanülierung verbunden sind, unbedingt im stationären Setting getragen werden und ggf. durch eine Probedekanülierung verringert werden.

Ein weiterer wichtiger Schritt wird die systematische Evaluation des vorgestellten Protokolls sein. Dies ermöglicht eine objektive Einschätzung des Behandlungserfolgs, der mit dem standardisierten interdisziplinären Dekanülierungsmanagement erzielt werden kann. Hier wird neben der Behandlungsdauer vor allem die Sicherheit des Patienten im Vordergrund stehen, d.h. die Frage, ob eine effektive und komplikationsfreie Dekanülierung des Patienten erreicht werden kann. Zurzeit erfassen wir Daten von Patienten, die mit dem hier vorgestellten Management dekanüliert worden sind, und bereiten eine solche systematische Evaluation vor. Auch das gesamte Vorgehenskonzept wird kontinuierlich weiterentwickelt und unseren klinischen Erfahrungen angepasst. Hier ist die Zusammenarbeit zwischen mehreren erfahrenen Therapeuten aus unterschiedlichen Kliniken von großer Bedeutung.

Die Behandlung tracheotomierter Patienten erfordert standardisierte Vorgehensweisen, die ein einheitliches Vorgehen im interdisziplinären Team gewährleisten und so zu einer verbesserten Behandlungseffizienz aber auch zu einer erhöhten Sicherheit des Patienten im Dekanülierungsprozess führen. Solche Behandlungsschemata sollten jedoch einen dynamischen Charakter haben und sich an neue Erfahrungen und veränderte klinische Bedingungen jederzeit anpassen lassen.

Interessenkonflikt: Die Autoren geben an, dass kein Interessenskonflikte besteht. Abb. 2 wurde mit freundlicher Genehmigung von Andreas Fahl Medizintechnik-Vertrieb GmbH, Köln, den Autoren zur Veröffentlichung überlassen.

\section{Kernbotschaft}

Bei tracheotomierten Patienten, die komplexe respiratorische und schluckphysiologische Defizite aufweisen, kann im Rahmen eines Probedekanülierungsversuchs mit Verwendung eines Platzhalters eingeschätzt werden, ob eine dauerhafte Dekanülierung möglich ist oder nicht. Hierzu werden Evaluationskriterien und das Ablaufprotokoll vorgestellt. 


\section{Literatur}

1 Elpern EH, Scott MG, Petro L et al. Pulmonary aspiration in mechanically ventilated patients with tracheostomies. Chest 1994; 105: 563-566

2 Olep K, Leonard Getch K, Criner GJ. Swallowing dysfunction in patients receiving prolonged mechanical ventilation. Chest 1996; 109: 167-172

3 Ding $R$, Logemann JA. Swallow physiology in patients with trach cuff inflated or deflated: a retrospective study. Head Neck 2005; 27: 809-813

4 Leder SB, Ross DA. Confirmation of no causal relationship between tracheotomy and aspiration status: a direct replication study. Dysphagia 2010; 25: 35-39

5 Terk AR, Leder SB, Burrell MI. Hyoid bone and laryngeal movement dependent upon presence of a tracheotomy tube. Dysphagia 2007; 22: 93-98

6 Winklmaier $U$, Wüst K, Schiller $S$ et al. Leaking of fluid in different types of tracheal tubes. Dysphagia 2006; 21: 237-242

7 Frank U, Mäder M, Sticher H. Dysphagic patients with tracheotomies: a multidisciplinary approach to treatment and decannulation management. Dysphagia 2007; 22: 20-29

8 Schlaegel W. Das Trachealkanülenmanagement in der neurologischen Frührehabilitation. Neuro Rehabil 2009; 15: 301-307

9 Schlaegel W. Poststationärer Verlauf schluckgestörter Patienten mit Hirnschädigungen - 5-Jahres-Follow-up-Studie. Neuro Rehabil 2010; 16: 131-136 